\title{
Systematic Analysis and Quality Evaluation for Pharmacoeconomic Studies on Traditional Chinese Medicines
}

Nan Yang

Sichuan University

Huihui Zhang

Sichuan University

Taoyi Deng

Sichuan University

Ming Hu ( $\nabla$ huming_@163.com )

Sichuan University https://orcid.org/0000-0002-3637-4130

\section{Research}

Keywords: Traditional Chinese Medicine, Pharmacoeconomics, System Review, Quality Evaluation

Posted Date: April 6th, 2020

DOI: https://doi.org/10.21203/rs.3.rs-18494/v1

License: (9) This work is licensed under a Creative Commons Attribution 4.0 International License. Read Full License 


\section{Abstract}

Background: This study aimed to describe the current situation of the published Pharmacoeconomic researches on Traditional Chinese Medicine and evaluate the quality of the researches to analyze existing problem and make suggestions for improvement.

Methods: We developed a search strategy, set inclusion and exclusion criteria and searched the articles of Pharmacoecnomic studies on Traditional Chinese Medicine from CNKI, VIP, Wanfang, Sinomed database and Pubmed from their inception to February 2020. Basic information of the literatures was extracted. Then quality of each article was evaluated by using Guidelines for authors and peer reviewers of economic submissions to the BMJ.

Results: 430 articles with 433 pharmacoeconomics studies were included. And the information of time of publication, journal, author, funding, diseases, interventions, research design, evaluation methods, cost identification, health outcomes, uncertainty analysis and so on. The overall quality score of the literatures was 0.62 according to the evaluation standard of BMJ guidelines. The highest mark was 0.85 and the lowest mark was 0.38 .

Conclusions: Quality of pharmacoeconomic studies on traditional Chinese medicines was generally low.There were some problems in the published pharmacoeconomic articles, such as lack of fund support, short research horizon, unclear perspective and research design, misidentification of cost, poor comparability of health outcome and so on. We suggested to develop guidelines for pharmacoeconomic evaluation on Traditional Chinese Medicine to standardize the research procedure and establish reasonable health outcome indicators of Traditional Chinese Medicine to enhance comparability and generalization of studies.

\section{Background}

Pharmacoeconomics is a complex science that provides evidences for the optimal allocation and efficient utilization of medicine resources. Since the 1970s, it has been gradually accepted and applied by health care providers, health policy makers, medical insurance institutions, etc., as the basis of a new drug application, drug pricing, drug purchasing of medical institutions, National Essential Medicines List formulation, medical insurance and post-marketing drug evaluation, etc ${ }^{[1-3]}$. Traditional Chinese Medicine (TCM) has been playing an important role in the field of medical treatment in China with Chinese patent medicine making up 38\% share of the drug market. In recent years, with the increasing attention to economic evaluation of medicines, there were more and more pharmacoeconomic researches that could comprehensively evaluate the effect and economy of TCM. However, due to data gathering, standardization of clinical research and so on, pharmacoeconomic researches on TCM were of variable quality. This study aimed to describe current situation, analysis the existing problems of pharmacoeconomic researches on TCM and make suggestions for further improvement of the pharmacoeconomic research on TCM. 


\section{Methods}

\section{Data sources}

We searched the following databases between their inception and February 2020: China National Knowledge Infrastructure(CNKI) (1974), Chongqing VIP Information(VIP) (1989), WANFANG Database(1998), Sinomed (1978), and Pubmed (1966).

The search strategy was ("Chinese medicine" OR "Traditional Chinese Medicine" OR "Chinese patent medicine" OR "herbal medicine" OR "natural medicine" OR "Chinese Medicinal Materials" OR "Botanical medicine" OR "Chinese herbal pieces" OR"Chinese medicinal granula") and ("pharmacoeconomics" OR "economics" OR "Cost Effectiveness Analysis" OR "Cost Utility Analysis" OR "Cost Benefit Analysis" OR "Cost Minimization Analysis" OR "Markov" OR "Decision Tree"). Chinese and English terms for search keywords were used when searching the databases. In addition, the references in all retrieved articles as well as our department files were searched.

\section{Study selection}

We included all pharmacoeconomics evalueation article on TCM. We excluded: (1) research on traditional Chinese treatment, like acupuncture, Guasha, massage, etc; (2) literatures of theory introduction, review, report, interview and comment of theoretical methods.

\section{Data extraction and quality assessment}

Hard copies of all articles included in the study were read in full independently by two authors $(\mathrm{HH}$ Zhang, TY Deng). Data from the articles were extracted according to pre-defined criteria. We designed data extraction table by using Excel software, and extracted information of article including title, first author, first author's affiliation, type of the first author's affiliation, location of the first author, journal, time of publication, disease of the study, sample size of study, age range of the sample, grouping, study design, medicines for intervention, cost identification, health outcomes, uncertainty analysis, perspective, evaluation techniques, funding and so on. Then we analyzed the extracted data statistically and systematically and evaluated the quality of literatures by using Guidelines for authors and peer reviewers of economic submissions to the BMJ ${ }^{[4]}$.

\section{Results}

\section{Study description}

According to the pre-determined search strategies and inclusion and exclusion criteria, the literatures were searched and screened (Fig. 1). We eventually included 424 articles in Chinese and 6 articles in English, which included 433 studies.

\section{Time of publication}


The first pharmacoeconomic study on TCM in Chinese was first appeared in Chinese Journal of Pharmacoepidemiology in 1997, which was released by Xia Lingsan and focused on Ahylysantinfarctase and Fufang Danshen injection for cerebral infarction patients ${ }^{[5]}$. Since 2002, the number of published pharmacoeconomic studies on TCM had gradually increased, with 45 articles published in 2017 at most (Figure. 2).

\section{Journals}

In addition to 25 dissertations, other pharmacoeconomic evaluation literatures on TCM were published in 172 domestic and foreign medical journals, among which 106 were Chinese core journals criterion, 4 were journals of Science Citation Index (SCl) and 133 other core journals. China Pharmacy (36 articles), China Journal of Pharmaceutical Economics (14 articles), Evaluation and Analysis of Drug-Use in Hospitals of China (13 articles), China Practical Medicine (12 articles), China Pharmaceuticals (12 articles), Strait Pharmaceutical Journal (12 articles), China Health Care Nutrition(10 articles), Chinese Journal of Pharmacoepidemiology (8 articles), Journal of Medical Theory and Practice (6 articles), Guide of China Medicine (6 articles) and Journal of Clinical Rational Drug Use ( 6 articles) published more articles in Chinese. Journals included in $\mathrm{SCl}$ published the articles in English were Journal of Alternative and Complementary Medicine (2 articles), Evidence Based Complement Alternative Medicine (2 articles), Trials ( 1 articles) and Chinese Journal of Integrative Medicine (1 articles).

\section{First authors and their affiliations}

More than $81.16 \%$ of the pharmacoeconomic evaluation literatures on TCM were carried out by the first author from medical institutions, and the rest literatures' first authors came from universities, research institutions or enterprises. The distribution of the first authors from medical institutions was dispersed. the first authors from universities was more concentrated in Beijing University of Chinese Medicine published 7 articles, Guangxi Medical University and China Pharmaceutical University published 6 articles respectively.

\section{Funding}

There were 364 literatures without indicating the source of funds (84.65\%), and only $66(15.35 \%)$ were marked with funding support, which included 9 state projects, 22 provincial and ministerial funded projects, 22 municipal funded projects, 4 University funding projects, 4 funding projects of the authors' units, 3 project supported by the foundation, and 2 Enterprise funded projects.

\section{Disease}

According to the International Classification of Diseases (ICD-10), the 430 published literatures ranged over 148 kinds of diseases' treatments, among which the top five kinds of disease included nervous 
system, cardiovascular system, respiratory system, gynecology and digestive system®as noted at Figure 3.

\section{Medicines for treatment}

The medicines treatment involved in the studies were complex and diverse, which included Chinese medicine v.s. Chinese medicine (164 studies), Chinese medicine v.s. Western Medicine (170 studies); the comparison of combined drug schemes includes Chinese medicine plus western medicine v.s. Western Medicine (76 studies), Chinese medircine plus western medicine v.s. Chinese medicine plus western medicine (20 studies), and the evaluation of Chinese medicine prescriptions (15 studies), Chinese medicine plus western medicine v.s. Chinese medicine (3 studies).

There were 262 kinds of Chinese patent medicines and 70 kinds of traditional Chinese medicine prescriptions (such as prescription, pieces decoction, powder, extraction, tincture, pills) in the studies. In 433 studies, the most Chinese patent medicines studied were Xuesaitong Tablet (20 studies), Fufang Danshen Tablet / Dropping Pill (17 studies), Shuxuening Injection ( 16 studies), Danhong Injection (15 studies), Qingkailing Injection (15 studies), Shuxuetong Injection (13 studies), Xiyanping Injection ( 13 studies), Fufang Danshen Injection (12 studies), Danshen Chuanxiongqin Injection (12 studies), Ciwujia Injection (11 studies), Shenmai Injection (10 studies), Xueshuantong Injection (10 studies), Tanreqing Injection ( 9 studies), Yinxingdamo Injection ( 9 studies), Yanhuning Injection ( 9 studies), Aidi injection ( 8 studies), Reduning Injection (8 studies), Rupixiao tablet (12 studies).

\section{study design and comparators}

Study design used in the 433 studies included randomized clinical intervention (235 studies), retrospective study (162 studies), prospective study (12 studies) and decision tree model (16 studies). In addition to 12 studies without marking the research time horizon, and 13 studies that got parameters through literature or model simulation, other studies' research time horizon ranged from half a day to two years (Table 1).

Table 1. Research Time Horizon

\begin{tabular}{ccc}
\hline research time & Numbers of study & Percentage \\
\hline Less than $14 \mathrm{~d}$ & 158 & $41.62 \%$ \\
$15 \mathrm{~d}$ to $30 \mathrm{~d}$ & 100 & $26.18 \%$ \\
$1 \mathrm{~m}$ to $3 \mathrm{~m}$ & 99 & $25.92 \%$ \\
$4 \mathrm{~m}$ to $6 \mathrm{~m}$ & 11 & $2.88 \%$ \\
$7 \mathrm{~m}$ to $12 \mathrm{~m}$ & 9 & $2.36 \%$ \\
$12 \mathrm{~m}$ to $24 \mathrm{~m}$ & 4 & $1.04 \%$ \\
\hline
\end{tabular}

Note: "d" represents day, "m" represents month 
Nearly half studies compared between two interventions; other half compared three and four interventions; a few other studies compared more than five interventions.186 studies did not describe the reason for the comparators.

\section{Sample size and perspective}

Expect 21 studies extracting parameters from other literatures, the sample size of other studies ranged from 38 to 2820 subjects, which was shown in Figure 4. The age of subjects was from 0 to 90 years old. $376(86.84 \%)$ studies did not clearly define the perspective of their pharmacoeconomic evaluations. 24 of the remaining studies were from the perspective of medical institutions, 13 from the patients, and 11 from the whole society, 11 from the payers, 7 from the patients, and 2 from health system.

\section{Evaluation techniques}

More than $82 \%$ of the studies chose cost-effectiveness analysis (CEA), and the others study used at least one method of cost analysis, such as Cost Minimization Analysis (CMA), Cost Benefit Analysis (CBA), and Cost Effectiveness Analysis (CUA). In the 28 studies, the above methods were used in combination with the Decision Tree (DT) model for short-term predictions or Markov model for long-term predictions (Table 2).

Table 2 Evaluation Techniques Details

\begin{tabular}{|c|c|c|}
\hline Evaluation Methods & Numbers of Studies & Percentage \\
\hline CEA & 354 & $81.76 \%$ \\
\hline $\mathrm{CEA}+\mathrm{DT}$ & 18 & $4.16 \%$ \\
\hline CMA & 16 & $3.70 \%$ \\
\hline $\mathrm{CEA}+\mathrm{CUA}$ & 15 & $3.46 \%$ \\
\hline $\mathrm{CEA}+\mathrm{CMA}$ & 10 & $2.31 \%$ \\
\hline CBA & 7 & $1.62 \%$ \\
\hline CUA & 3 & $0.67 \%$ \\
\hline $\mathrm{CMA}+\mathrm{DT}$ & 3 & $0.69 \%$ \\
\hline CUA+Markov model & 3 & $0.69 \%$ \\
\hline CEA+Markov model & 3 & $0.69 \%$ \\
\hline $\mathrm{CUA}+\mathrm{DT}$ & 1 & $0.23 \%$ \\
\hline
\end{tabular}




\section{Cost scope and identification}

Costs of those pharmacoeconomic evaluation studies could be generally classified as direct cost and indirect cost. 356 studies (83.22\%) integrated direct cost into the studies; 50 studies (11.55\%) integrated direct cost and indirect cost into the studies; 27 studies $(6.24 \%)$ did not clearly define their cost scope.

Table 3. Cost Identification

\begin{tabular}{ccc}
\hline Cost & Numbers of Studies & Percentage \\
\hline direct cost & 356 & $82.22 \%$ \\
direct cost + indirect cost & 50 & $11.55 \%$ \\
Not clearly define & & \\
& 27 & $6.24 \%$ \\
\hline
\end{tabular}

Direct costs in the studies and frequency of respective occurrence were drug fee (384 studies), examination fee (157 studies), treatment fee (78 studies), hospitalization fee (42 studies), nursing fee (42 studies), bed fee (41 studies), registration fee (31 studies), dispensing fee (27 studies), adverse reaction treatment fee (23 studies), material fee (16 studies), meal fee (11 studies), surgery fee (9 studies), disposal cost ( 6 studies), consultation fee ( 1 studies), blood transfusion fee (1 studies), instrument loss (1 studies) and other expenses (54 studies). Indirect costs in the studies and frequency of respective occurrence were time cost (22 studies), compensation (fee) for one's absence from work (18 studies), labor loss (3 studies), and death cost (1 studies).

\section{Health outcomes}

Among 433 pharmacoeconomic evaluation studies, 225 studies used single end-point, 156 studies used double end-points, 26 studies used three end-points, and the other 26 studies used four or more endpoints at the same time.

$90.76 \%$ (393) studies used the final end-points as the health outcomes, which specifically included clinical efficiency/improvement rate (263 times), adverse reactions rate (132 times), efficiency rate of TCM syndromes (47 times), recurrence rate (11 times), and disease or drug withdrawal rate (16 times), death rate (5 times), Quality-adjusted Life Year (18 times). 14.09\% (61) studies used the intermediate endpoints as the health outcomes, which included score/index scale (41 times), biochemical indicators (22 
times), physiological indices (9 times), and imaging indices (9 times). 11 studies (2.5\%) used health utility as the health outcomes. One studies used net benefit as the health outcomes.

\section{Uncertainty analysis and generalizability}

$66.51 \%$ the studies (288) carried out uncertainty analysis, and the main uncertainty analysis variables were shown in Table 4. Uncertainty analysis of 219 (76.04\%) studies were assessed by using one-way sensitivity analysis with reducing drug prices by 10\% - 20\%. 69 (23.96\%) studies used multi-way sensitivity analysis for uncertainty.

Table 4. Uncertainty analysis variables of the studies

\begin{tabular}{|c|c|c|}
\hline Uncertainty analysis & studies & percentage \\
\hline drug prices & 190 & $65.97 \%$ \\
\hline $\begin{array}{l}\text { drug prices and other cost (such as examination fee, examination fee, bed fee, treatment fee, nursing fee, compensation } \\
\text { (fee) for one's absence from work etc.) }\end{array}$ & 67 & $23.26 \%$ \\
\hline drug prices and health outcome (such as efficiency rate etc.) & 19 & $6.06 \%$ \\
\hline $\begin{array}{l}\text { other cost (such as examination fee, examination fee, bed fee, treatment fee, nursing fee, compensation for one's } \\
\text { absence from work etc.) }\end{array}$ & 7 & $2.43 \%$ \\
\hline Other variables (such as course of medication, discount rate) & 5 & $1.74 \%$ \\
\hline
\end{tabular}

101 articles (23.33\%) discussed the limitations of the results, and 12 articles discussed the generalizability of the results. Although 66 studies took children under 18 as the sample, none of them made mention of the ethical issues of the study.

\section{Quality assessment of pharmacoeconomic evaluation literatures on TCM}

In this study, we used guideline for authors and peer reviewers of economic submissions to the BMJ, developed by Drummond M.F. and Jefferson T.O. to evaluate the quality of the literature. This guideline set up 35 evaluation items with 10 sections under three headings including study design, data collection, and analysis and interpretation of results. In this study, the following scoring rules were set up.

If the evaluation result of each item was marked as "yes", 1 point would be scored. If the evaluation result is marked as "no" or "unclear", 0 point would be scored.

Each study was scored based on the proportion of items it should have met according to the guideline of $\mathrm{BMJ}$, the maximum score of which was 1 . The overall quality score of a pharmacoeconomics evaluation researches on TCM was the average score of each study. If the score is greater than 0.90 , it indicated that the literature quality was high ${ }^{[6]}$. And we also calculated the score of every item in guideline of BMJ. The specific quality evaluation scoring results were shown in Table 6. 
Table 6 Quality Evaluation Scoring of Pharmacoeconomic Research on TCM 
Study Question

Form of Evaluation

Effectiveness Data

Benefit Measurement and

Valuation

Costing

Modelling

Adjustments for Timing of Costs

and Benefits

Presentation of Results
The research question was stated

The economic importance of the research question was stated

The viewpoint(s) of the analysis were clearly stated and justified

The rationale for choosing the alternative programmes or interventions compared was stated

The alternatives being compared were clearly described

The form of economic evaluation used was stated

The choice of form of economic evaluation was justified in relation to the questions addressed

\section{Data collection}

The source(s) of effectiveness estimates used were stated

Details of the design and results of effectiveness study were given (if based on a single study)

Details of the method of synthesis or meta-analysis of estimates were given (if based on an overview of

a number of effectiveness studies)

The primary outcome measure(s) for the economic evaluation were clearly stated

Methods to value health states and other benefits were stated

Details of the subjects from whom valuations were obtained were given

Productivity changes (if included) were reported separately

The relevance of productivity changes to the study question was discussed

Quantities of resources were reported separately from their unit costs

Methods for the estimation of quantities and unit costs were described

Currency and price data were recorded

Details of currency of price adjustments for inflation or currency conversion were given

Details of any model used were given

The choice of model used and the key parameters on which it was based were justified

\section{Analysis and interpretation of results}

Time horizon of costs and benefits was stated

The discount rate(s) was stated

The choice of rate(s) was justified

An explanation is given if costs or benefits were not discounted

Details of statistical tests and confidence intervals were given for stochastic data

The approach to sensitivity analysis was given

The choice of variables for sensitivity analysis was justified

The ranges over which the variables were varied are stated

Relevant alternatives were compared

Incremental analysis was reported

Major outcomes are presented in a dissaggregated as well as aggregated form

$\begin{array}{lll}433 & 0 & 1 \\ 313 & 120 & 0.723 \\ 62 & 371 & 0.143 \\ 243 & 190 & 0.561 \\ 391 & 42 & 0.903 \\ 433 & 0 & 1 \\ 433 & 0 & 1\end{array}$

220

213

0.508

260

58

0.818

3

0.880

433

432

0.002

$397 \quad 36$

0.917

389

0.102

376

0.132

2

408

431

0.005

25

0.942

422

11

0.975

24

433

0

0.857

0.929

$$
2
$$

432

10

10

10

18

28

288

288

433

273

433
0.998

0.023

0.023

0.042

0.065

0.665

0.665

0.663

1

0.837

Page $10 / 20$ 
The results showed that the overall quality score of 433 pharmacoeconomics studies on TCM was 0.62 according to the evaluation standard of BMJ guidelines. The highest mark was 0.85 and the lowest mark was 0.38 . Scores of majority studies were concentrated in $0.5-0.7$. The distribution was showed in Table 7 ranking according to marks.

Table 7. Distribution of score of pharmacoeconomics studies on TCM

\begin{tabular}{ccc}
\hline Score & Number of studies & Percentage \\
\hline $0.3-0.4$ & 1 & $0.23 \%$ \\
$0.4-0.5$ & 19 & $4.39 \%$ \\
$0.5-0.6$ & 147 & $33.95 \%$ \\
$0.6-0.7$ & 200 & $46.19 \%$ \\
$0.7-0.8$ & 62 & $14.32 \%$ \\
$0.8-0.9$ & 4 & $0.92 \%$ \\
\hline
\end{tabular}

Among them, items of "stating methods to value health states and other benefits", "reporting quantities and their unit costs of resources separately", "adjustment details of currency of prices for inflation or currency conversion", "the choice of rate(s)", "the discount rate(s)", "an explanation without being discounted", and "details of statistical tests and confidence intervals for stochastic data" scored below 0.1. Items of "perspective/viewpoints", "productivity change reporting", "Time horizon", and "conclusions accompanied by the appropriate caveats" also scored low. And items on the rationale stating for choosing the alternative programmes or interventions compared, source(s) stating of effectiveness estimates did not score high.

When calculating separately, the average quality score of the 6 literatures in English was 0.73 . The results showed better quality of pharmacoeconomics research literatures on TCM from SCI journals, especially in the items of research perspective and discounting. However, uncertainty analysis and productivity measurement of the study remained to be improved.

\section{Discussions}

At present, the published pharmacoeconomic studies on TCM were in small number of sample size, conducted by numerous research institutions and lack of fund support. In the past decade, the number of the published pharmacoeconomic research articles on TCM had being increasing annually, but the total was less than one tenth of the number of published pharmacoeconomic research articles on chemical drugs. Moreover, researchers of those studies were mostly from various medical institutions, which were decentralized and only $15.35 \%$ studies had funding support. Chinese patent medicines had rose to 
49.98\% of all drugs listed in National Drug Catalogue for Basic Medical Insurance of China, which was released in $2019^{[7]}$. Although the government had considered the factor from pharmacoeconomics evaluation on TCM in the policy design of medical insurance reimbursement, governmental drug procurement and pricing, it was not clear how much current published studies were worth.

Overall quality of published pharmacoeconomic research articles on TCM was low. Although nearly half of the studies were published in Chinese core journals criterion and even journals of Science Citation Index, compared with the study of Sun ${ }^{[1]}$ in 2015 , the quality of pharmacoeconomics research articles on TCM has not improved in recent five years; compared with the studies of Chen ${ }^{[8]}, \mathrm{Li}^{[9]}, \mathrm{Lei}^{[10]}$ in 2004 , 2008 and 2010 respectively, the quality of pharmacoeconomics research articles on TCM was lower than that on chemical drugs. On the one hand, none single consistent guideline for TCM pharmacoeconomic evaluation has been accepted by the industry, and there may be some dislocation and bias when using pharmacoeconomic evaluation guideline on chemical drugs for TCM. On the other hand, the researchers of pharmacoeconomics evaluation research on TCM mostly came from medical institutions, were lack of thorough understanding of the basic theory of pharmacoeconomic, which led to poor quality of overall researches.

Most researches did not clearly described perspectives and study design of the researches lacked scientific basis. The perspective of pharmacoeconomics evaluation would affect cos identification and measurement, the selection and calculation of health outcomes and be consistent in a study ${ }^{[11]}$, however, more than $85 \%$ of the studies did not mention their research perspectives, which had negative impact on the integrity and reliability of the study. The sample size of 433 studies ranged from dozens to thousands, with great differences. Although the China guideline for pharmacoeconomic evaluation suggested the sample size should meet the needs of different pharmacoeconomics evaluation ${ }^{[11]}$, there was only four studies describing the process of sample size estimation. More than half of the studies reported randomized controlled trials, but the vast majority of the studies did not provide specific methods for randomization. Moreover, some studies obviously confused retrospective cohort study, mixed study and randomized clinical intervention study. In terms of control group, nearly half of the studies did not explain the reasons of setting. If researchers only compare several interventions at will, it would be difficult to ensure the generalizability and application of the results.

Some studies included identified and measured cost wrongly and health outcomes in different studies had poor comparability. The majority of studies only calculated the direct cost, or even only the drug cost, which did not fully reflect the cost of treatments. A small number of studies measured both direct and indirect costs, and still some studies did not mention the issue of cost measurement. These showed that researchers had misunderstood or confused the definition and identification of cost in pharmacoeconomic evaluation, which lead to the problem of calculating cos repeatedly or the absence of complete cost. The prescription and using of TCM under the diagnosis, treatment and syndrome differentiation method of traditional theory have unique characteristics compared with chemical medicine, which have unique experience and advantages in improving the rehabilitation effect 
and health utility of patients through holistic treatment. However, only $13.70 \%$ studies of pharmacoeconomic evaluation on TCM used Chinese medicine syndrome as outcome, and only $3.21 \%$ studies used health utility indicator. Instead, clinical efficiency and adverse reactions were used most frequently. This may occur in response to lack of standardized and quantifiable evaluation indicators of TCM symptoms and effects.

Time horizon of the studies was commonly short. Nearly $70 \%$ studies were within one month and only $1.04 \%$ studies lasted more than one year. This might be why only $2.3 \%$ costs in the literatures were discounted. Except a few studies on acute diseases, most studies on chronic diseases with relatively long treatment cycle. However, these studies did not fully reflect the advantages of TCM in improving longterm clinical efficacy, reducing recurrence rate and treatment costs.

\section{Conclusions}

Quality of pharmacoeconomic studies on traditional Chinese medicines was generally low, which was consistent with the results of previous quality assessment of economic evaluation on complementary and integrative medicine ${ }^{[12]}$.

We suggested researchers to formulate pharmacoeconomic evaluation guidelines being suitable for the characteristics of TCM and standardize the research procedure, such as complex prescription and excessive confounding factors. In 2013, Xie et al, published 'technical specification for pharmacoeconomic evaluation on post marketed Chinese patent medicine (Draft)' in China Journal of traditional Chinese Medicine ${ }^{[13]}$; and in 2019, Gao et al, published 'Guidelines for Quality Assessment of Pharmacoeconomic Evaluation Report' as group standardization ${ }^{[14]}$. Those guidelines considered the basic ideology and characteristics of traditional Chinese medicine to certain extent, however, they did not solute the specific problems faced by the pharmacoeconomic evaluation of TCM, such as how to map the effectiveness and health outcome of TCM and chemical medicines. Therefore, a scientific and unified pharmacoeconomic evaluation guideline for TCM should be performed combined with expert opinions, focusing on the characteristics and current research situation of TCM. Reasonable and operable requirements and suggestions should be put forward from the aspects of sample size, study design, perspective stating, intervention selection, evaluation method selection, cost identification, health outcomes determination, uncertainty analysis, report form, etc., so as to standardize research procedure and improve the quality and reliability of results.

Reasonable health outcome index for TCM should be establish to enhance clinical comparability. Traditional Chinese medicine takes holistic treatment as main concept, so it's better to evaluate overall outcome of TCM by using health utility as its clinical output index ${ }^{[15]}$. It is difficult to evaluate the utility of TCM due to lack of health-related quality of life scale of traditional Chinese medicine in China. Therefore, it is necessary to establish an objective, scientific and quantifiable evaluation system of TCM effect indicators. On the one hand, there is need to establish the judgment standard of Chinese Medicine syndrome efficacy generally accepted to reduce the bias of subjective factors ${ }^{[15]}$. On the other hand, 
researchers should explore the corresponding relationship between symptoms, syndrome types of Chinese Medicine and disease diagnosis and classification of Western Medicine, so as to enhance the comparability between different treatments, expand the clinical applicability and generalizability for government's decision-making of the results of pharmacoeconomics research on TCM.

Strengthening cooperation of clinical and academic insititution might be helping to improve research quality. The number of pharmacoeconomic studies on TCM has been increasing in the past 40 years, but most clinicians and health policy makers remain skeptical of the results ${ }^{[16]}$.

Many pharmacoeconomics researchers also found that the research quality was generally low when they evaluated the published literature. There were many problems in the selection of interventions and comparators, study design, cost identification, result expression and uncertainty analysis. It is suggested that researchers with solid basic theoretical knowledge and clinical practitioners with rich research resources of pharmacoeconomics should strengthen cooperation, improve the level of research, and provide more reliable evidence for clinical treatment and health management decision-making.

\section{Abbreviations}

TCM: Traditional Chinese medicine; CNKI: China national knowledge infrastructure; VIP: Chongqing VIP Information; CEA: Cost-Effectiveness Analysis; CMA: Cost Analysis; CBA: Cost-Benefit Analysis; CUA: CostEffectiveness Analysis; DT: Decision Tree

\section{Declarations}

\section{Ethics approval and consent to participate}

Not applicable.

\section{Consent for publication}

Not applicable.

\section{Availability of data and materials}

All data are fully available without restriction.

\section{Competing interests}

The authors declare that they have no competing interests.

\section{Funding}

This research has been supported by National Key R\&D Program of China (Grant No. 2019YFC1709804), Sichuan Administration of Traditional Chinese Medicine (Project Number: 2018ZC004) and Sichuan 
Medical Law Research Center (Project Number: YF17-Y25) for their support in this research. They will not be involved to the analysis and interpretation of data, or make decision to submit the results.

\section{Authors' contributions}

HM and YN conceived the study design. ZHH and DTY searched and selected the articles, extracted, analyzed and interpreted the data. YN drafted the manuscript. HM critically reviewed the manuscript. All authors read and approved the final version of the manuscript.

\section{Acknowledgements}

We thank all the researchers who were engaged in pharmacoeconomic evaluation of TCM.

\section{Authors' information (optional)}

Ming Hu (Corresponding author): West China School of Pharmacy, Sichuan University, address at: No.17 People's South Road, Chengdu, China, E-mail address: huming_@163.com

Nan Yang: West China School of Pharmacy, Sichuan University, yoland62@163.com;

Huihui Zhang: West China School of Pharmacy, Sichuan University, 2274119159@qq.com

Taoyi Deng: West China School of Pharmacy, Sichuan University, taoyia1109@163.com

\section{References}

1. Sun X, Guo LP, Shang HC, Ren M, Lei X: Systematic economic assessment and quality evaluation for traditional Chinese medicines. China Journal of Chinese Materia Medica 2015, 40:2050-2053.

2. Zhang HB: Systematic evaluation of domestic pharmacoeconomics research literature in recent years. Chinese Licensed Pharmacist 2010,7:23-26.

3. National Healthcare Security Administration. Announcement on the release of the work plan for the adjustment of the national medical insurance drug catalogue in 2019. http://www.nhsa.gov.cn/art/2019/4/17/art_37_1214.html. [accessed on 10-3-2020]

4. Drummond M FQJefferson T O: Guidelines for authors and peer reviewers of economic submissions to the BMJ economic evaluation working party. BMJ 1996,313(7052):275-283

5. Xia LS, Wang R, Yang ZM: Pharmacoeconomic analysis of four treatments for cerebral infarction. Chinese Journal of Pharmacoepidemiology 1997,6: 167

6. Sara HD囚Nick B: The feasibility of creating a checklist for the assessment of methodological quality both of randomised and non-randomised studies of health care intervention区Epidemiol Community Health 1988,52: 377.

7. National Healthcare Security Administration. Notice on printing and distributing the catalogue of drugs for national basic medical insurance, work injury insurance and maternity insurance. 
http://www.nhsa.gov.cn/art/2019/8/20/art_37_1666.html.[accessed on 10-3-2020]

8. Chen W, Gao JM, Bi KN, Yang L: Systematic Review of Chinese Pharmacoeconomic Evaluation Literature. China Pharmacy 2004,15:28

9. Li MH, Li HC, Ma AX. Pharmacoeconomic Evaluation Studies in China: Status Quo, Problems and Suggestions. China Pharmacy 2008,19: 801-805.

10. Lei L, Hu M, Zhang Z: Systematic evaluation and quality evaluation of domestic pharmacoeconomic evaluation literatures in 2010. China Pharmacy 2014,24(26):2401-2404

11. Pharmacoeconomics Committee of China Pharmaceutical Association. China Guidelines for Pharmacoeconomics Evaluation[R]. 2019-07

12. Herman $P M$, Poindexter $B L$, Witt $C M$, et al: Are complementary therapies and integrative care costeffective? A systematic review of economic evaluations. BMJ Open 2012, 2(5):e001046-e001046.

13. Xie YM, Wang $X$, Wang N, Chang YP. Technical specifications for post-marketing pharmacoeconomic evaluation of Chinese medicine (draft version for comments). China Journal of Chinese Materia Medica 2013.18 $\ 2925-2929$.

14. Gao P, Fu XJ, Sun M, Kong C, Liang MH, Hao GL, Zhu WT: Exploration of Some Issues of Application Research on the Domestic Pharmacoeconomics. China Pharmacy 2014.25:481-482

15. Li MH, Liu GE. Significance and characteristics of economic evaluation of traditional Chinese medicine. China Journal of Pharmaceutical Economics.2009.3:11-14

16. Wu HY $\triangle T$ IAN CL. Quality Analysis of Pharmacoeconomics Evaluation and Application Literature in China during 2009-2013. China Pharmacy 2015.26 (11):1441-1444.

\section{Tables}

Table 5 was omitted by the authors in this version of the paper.

\section{Figures}




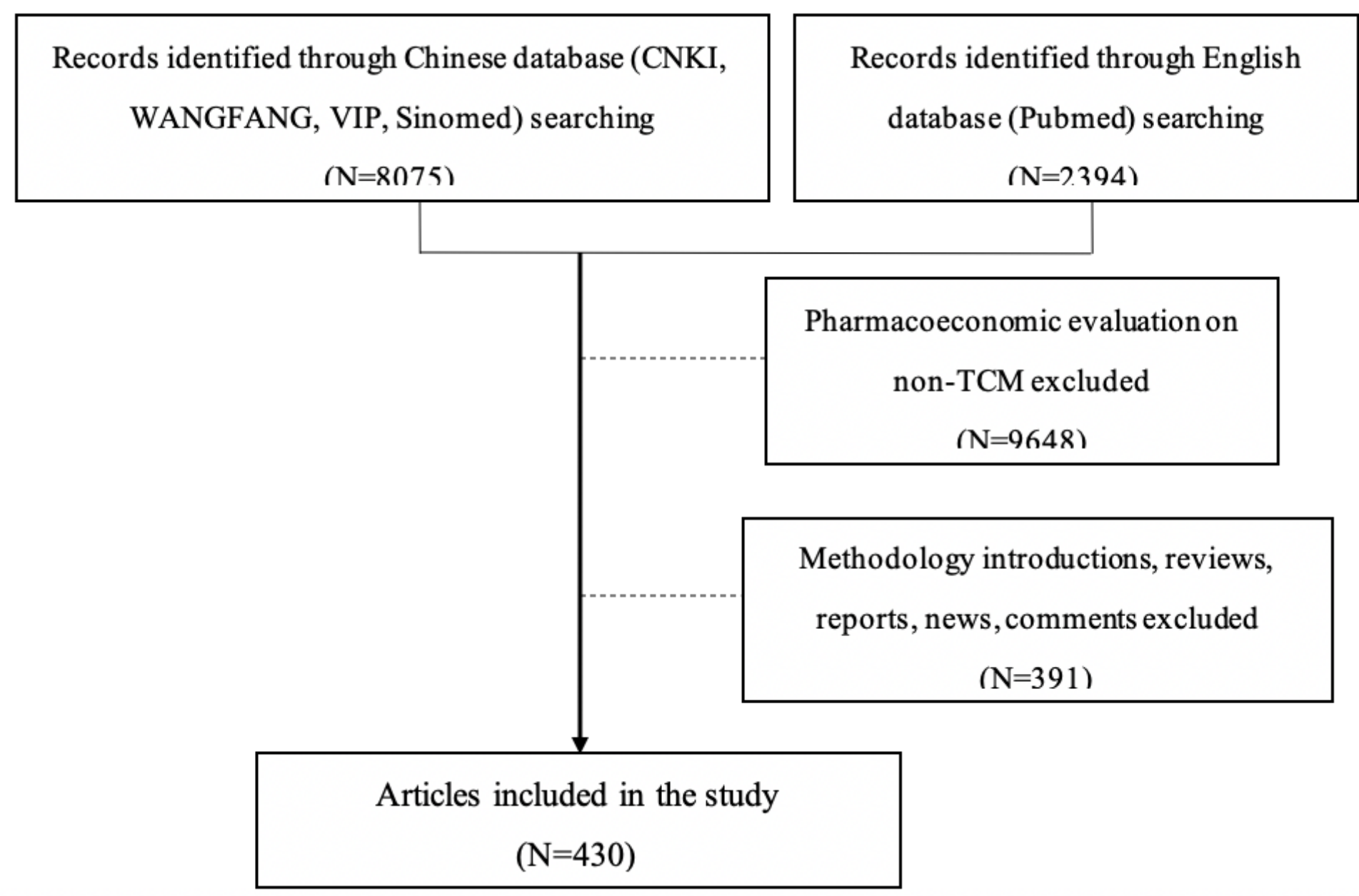

Figure 1

Flow Chart of Study Search and Selection 


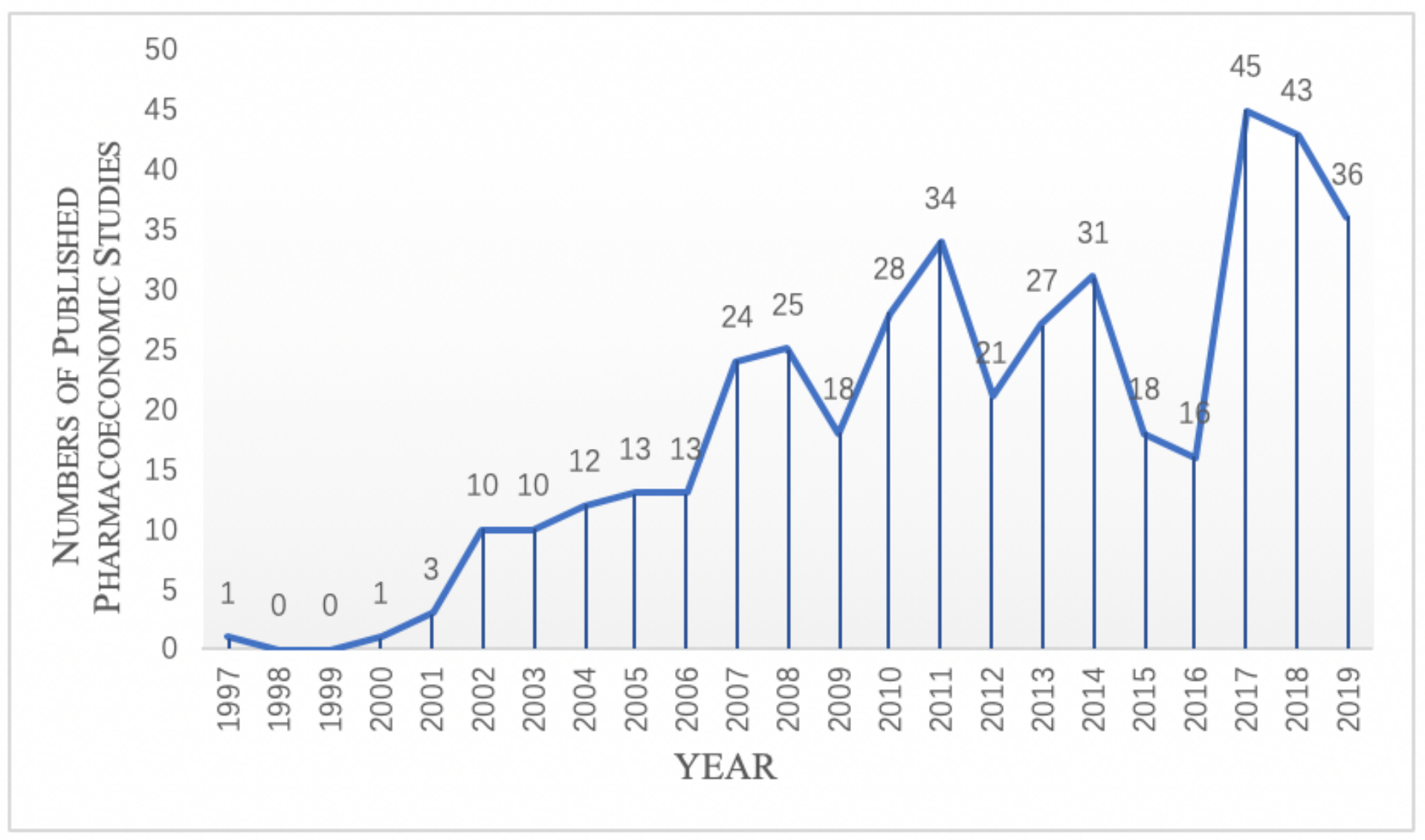

Figure 2

Time Distribution of Researches' Publication 


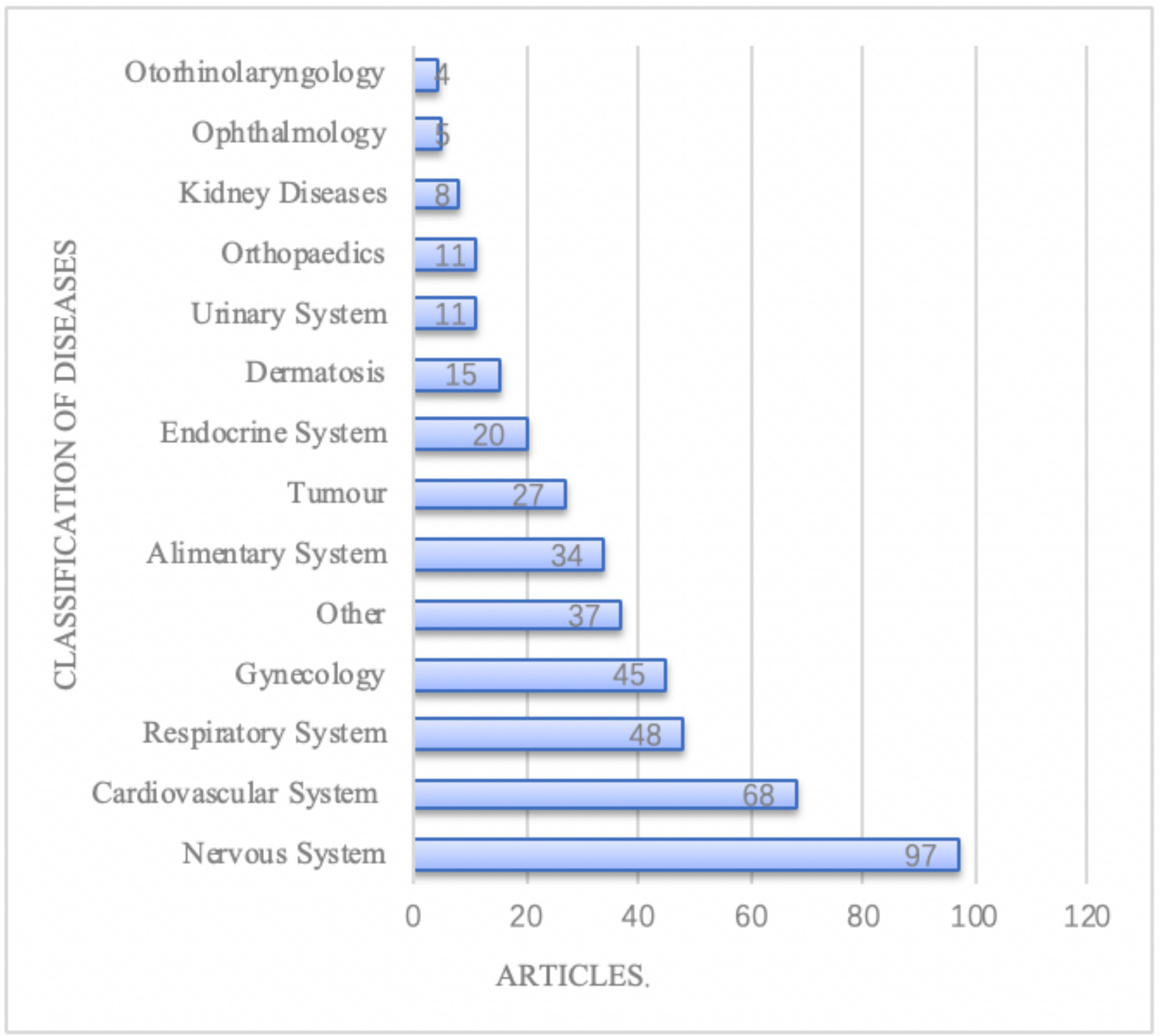

Figure 3

Disease Types of the Researches 


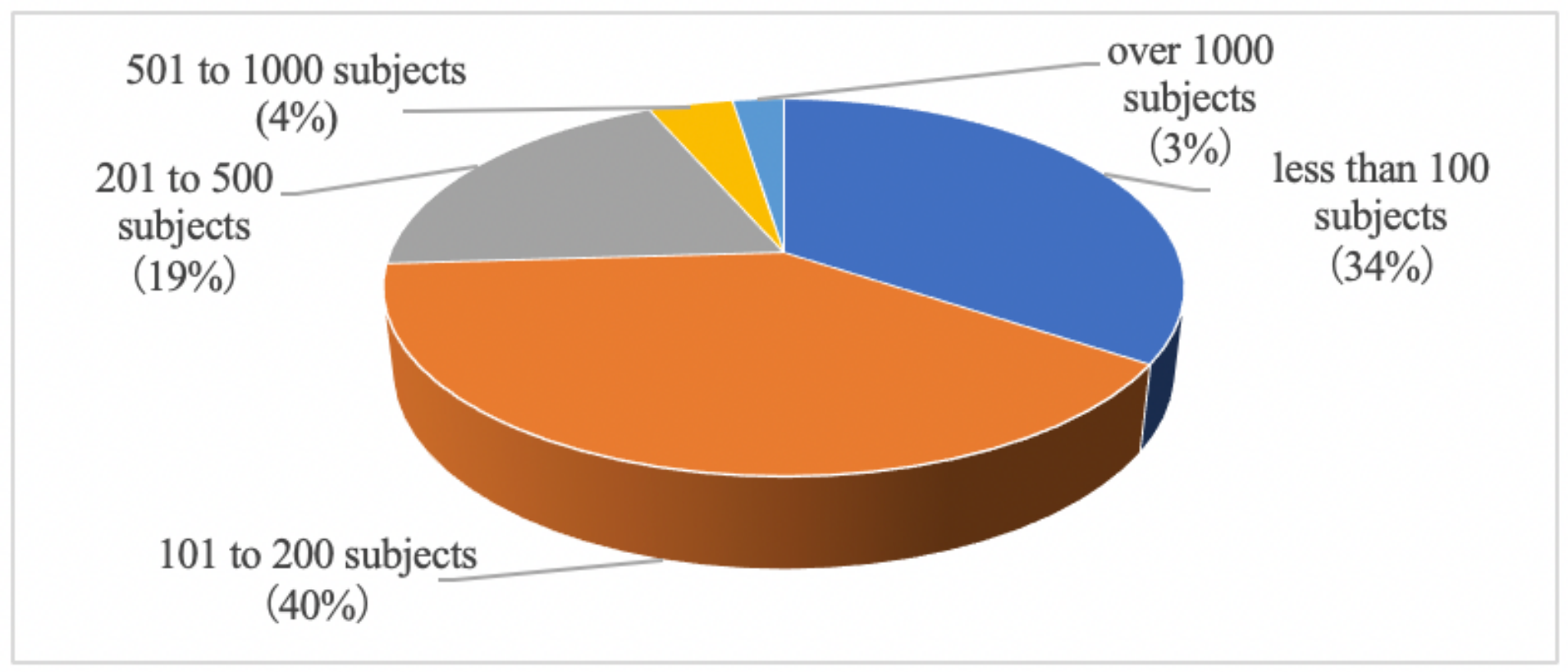

Figure 4

Distribution of Sample Size 